Classification

Physics Abstracts

$36.20 \mathrm{H}-72.20 \mathrm{~J}-72.40$

\title{
Effet des pièges sur la photoconductivité du polyacétylène partiellement isomérisé
}

\author{
G. Guillaud, M. Maitrot \\ Laboratoire de Physique Electronique 2, Bât. 203, Université Claude Bernard Lyon I, \\ 43, bd du 11 Novembre 1918, 69622 Villeurbanne Cedex, France \\ C. Mathis, B. François et J. J. André \\ Centre de Recherches sur les Macromolécules (C.N.R.S.), 6, rue Boussingault, \\ 67083 Strasbourg Cedex, France
}

(Reçu le 12 octobre 1983, accepté le 25 janvier 1984)

\begin{abstract}
Résumé. - Les photocourants transitoires induits par un flash lumineux ont été étudiés dans le domaine des temps longs $\left(10^{-3}-10^{2} \mathrm{~s}\right)$ sur le polyacétylène à différents taux d'isomérisation. Ces photocourants sont dominés par le piégeage et leur analyse permet le calcul du produit $\mu \tau$ et d'une constante de temps de dépiégeage $\left(\mu \tau=3 \times 10^{-11} \mathrm{~cm}^{2} / \mathrm{V}\right.$ et $\tau_{\mathrm{p}}=2 \times 10^{-3} \mathrm{~s}$ pour un échantillon essentiellement cis).

Abstract. - The transient photocurrents following a light flash are studied in the long time range $\left(10^{-3}-10^{2} \mathrm{~s}\right)$ on polyacetylene with different cis-trans isomerization ratios. These transient photocurrents are mostly related to trapping effects and their analysis allows the product $\mu \tau$ and the detrapping time constant $\left(\mu \tau=3 \times 10^{-11} \mathrm{~cm}^{2} / \mathrm{V}\right.$ and $\tau_{\mathrm{p}}=2 \times 10^{-3} \mathrm{~s}$ for a nearly cis sample) to be computed.
\end{abstract}

\section{Introduction.}

Les deux isomères du polyacétylène présentent une photoluminescence et des propriétés photoélectriques radicalement différentes [1-4]. Le cis- $(\mathrm{CH})_{x}$ possède une forte luminescence près du seuil de l'absorption interbande correspondant à la recombinaison rapide des porteurs de charge ; celle-ci est accompagnée d'une absence totale de photoconductivité. Au contraire le trans-(CH) $)_{x}$ est caractérisé par une importante photoconduction et une extinction de la luminescence. Cette différence de comportement est considérée comme une base expérimentale de la photogénération de paires de défauts chargés du type soliton/antisoliton dans le trans- $(\mathrm{CH})_{x}[1,5-7]$. De la nature des porteurs de charge va dépendre le mécanisme de transport dans les fibrilles.

Les études expérimentales des propriétés photoélectriques sont encore peu nombreuses et sont effectuées dans des conditions très diverses en utilisant soit une excitation laser pulsée soit une lumière modulée et une détection synchrone $[1-4,15]$. Il n'est pas certain que les mécanismes mis en jeu dans ces différents cas soient les mêmes. L'évolution dans le temps de la photoconduc- 
tivité du polyacétylène n'a fait l'objet que d'une étude dans le domaine $10^{-6}-10^{-3} \mathrm{~s}$ [2] et d'une étude aux temps très courts $\left(10^{-9}-10^{-8} \mathrm{~s}[15]\right)$.

Par une méthode déjà décrite [4] nous avons entrepris cette étude dans une gamme de temps plus longs $10^{-3}-10^{2} \mathrm{~s}$. Dans ce premier travail nous avons en particulier pu montrer de manière directe que les effets bolométriques n'apparaissent qu'à partir de temps d'irradiation voisin de $1 \mathrm{~s}$ et qu'ils sont négligeables pour les temps plus courts. Ceci justifie a posteriori des études effectuées à de faibles fréquences de modulation comme $13 \mathrm{~Hz}$ [2] ou $150 \mathrm{~Hz}$ [3]. De plus le comportement d'échantillons partiellement isomérisés indique une transition progressive entre le comportement du cis- $(\mathrm{CH})_{x}$ pur et celui du trans $-(\mathrm{CH})_{x}$ pur, faisant intervenir une participation croissante des pièges [4].

L'objet de cette lettre est l'étude de ces pièges par une analyse détaillée des photocourants transitoires. En effet l'étude de l'évolution dans le temps de la photoconductivité du polyacétylène permet de préciser un certain nombre de points et d'évaluer le produit $\mu \tau$ ( $\mu$ mobilité, $\tau$ durée de vie) ainsi que le taux de dépiégeage des porteurs. L'évolution de ces paramètres peut être suivie en fonction du pourcentage d'isomérisation cis-trans.

\section{Techniques expérimentales.}

Les échantillons étudiés ont été synthétisés selon la méthode développée par Shirakawa [8] et sont maintenus à $0^{\circ} \mathrm{C}$ dans des tubes scellés sous vide avant d'être utilisés. La concentration en impuretés a été testée par la méthode RBS [9]. Des électrodes en aluminium espacées de $1 \mathrm{~mm}$ ou de $0,5 \mathrm{~mm}$ sont évaporées sur une face du film sous une pression de $10^{-6}$ torr. Les échantillons sont alors transférés dans la cellule de mesure, la durée de ce transfert dans l'atmosphère ambiante étant inférieure à $2 \mathrm{~min}$.

La source lumineuse utilisée est une lampe à vapeur de xénon de $150 \mathrm{~W}$. Divers filtres interférentiels ou passe bande ainsi que des filtres neutres permettent de faire varier la longueur d'onde ou l'intensité. Les bandes utilisées sont centrées sur $3500 \AA$, sur $6250 \AA$ et sur $2 \mu$. Les énergies mesurées à l'aide d'une thermopile sont respectivement de 12,20 et $90 \mathrm{~mW} / \mathrm{cm}^{2}$.

Un disque à secteurs tournants génère des impulsions lumineuses de $3 \mathrm{~ms}$ de longueur et de fréquence voisine de $50 \mathrm{~Hz}$. Les courants sont mesurés à l'aide d'un amplificateur opérationnel LF 356, monté en ampèremètre différentiel.

\section{Résultats expérimentaux.}

Les courants transitoires induits par un flash lumineux ont une forme classique [10] (Fig. 1). L'augmentation rapide $\mathrm{AB}$ est due aux photoporteurs créés par la lumière selon un processus bande à bande. Elle est proportionnelle au flux lumineux et au champ électrique. Dans le cas d'échantillons essentiellement cis, la lumière UV est seule efficace. L'équilibre des populations de la bande de conduction et de la bande de valence est atteint en un temps très court. Ce temps correspond à la durée de vie $\tau$ des porteurs minoritaires créés par la lumière. C'est cette évolution qui a été étudiée par Etemad et al. [2] sur le trans-(CH)$)_{x}$. L'augmentation plus lente $\mathrm{BC}$ est due au piégeage de ces porteurs minoritaires, des porteurs de signe opposé entrant dans l'échantillon afin de maintenir la neutralité de celui-ci. Cette hypothèse implique que le courant dans le noir soit très supérieur à la variation dans la lumière. Ceci est toujours le cas pour les éclairements utilisés puisque $\Delta i / i$ est toujours inférieur à 0,1 .

Lorsque l'excitation lumineuse est supprimée, le courant chute brusquement CD d'une quantité approximativement égale à l'augmentation $\mathrm{AB}$. Ceci est dû à la recombinaison des porteurs en excès dans les bandes de conduction et de valence. Les porteurs piégés donnent par dépiégeage la variation plus lente au-delà de $\mathrm{D}$.

Ce schéma suppose que le taux de recombinaison bande à bande est supérieur au taux de piégeage et est donc valable en particulier pour les échantillons encore essentiellement $c i s$. 


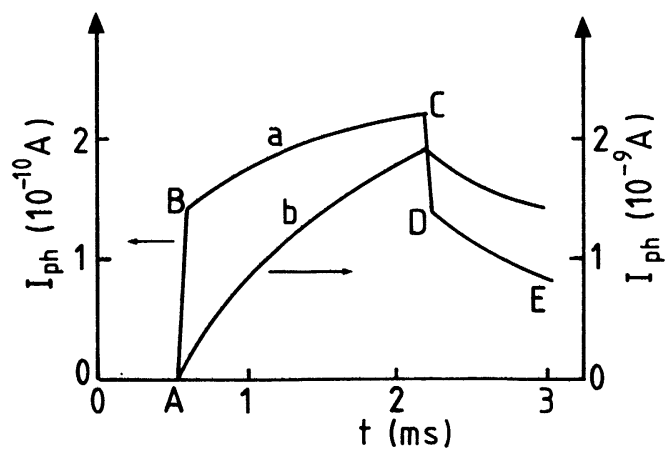

Fig. 1. - Photocourant transitoire induit par un flash lumineux de durée AC dans des films de polyacétylène cis (a) et trans (b). Les différentes phases de la réponse sont discutées dans le texte.

[Transient photocurrent initiated with a light flash of length AC in cis (a) and trans (b) polyacetylene films. The different steps of the response are discussed in the text.]

\section{Interprétation.}

Lorsque les porteurs minoritaires sont des électrons comme c'est le cas pour le polyacétylène, les équations régissant le processus de photoconduction ont la forme suivante :

$$
\begin{aligned}
& \frac{\mathrm{d} n}{\mathrm{~d} t}=l-r n+g n_{1}-n\left(N-n_{1}\right) S v \\
& \frac{\mathrm{d} n_{1}}{\mathrm{~d} t}=-g n_{1}+n\left(N-n_{1}\right) S v
\end{aligned}
$$

où $n$ et $n_{1}$ représentent la densité des électrons libres et des électrons piégés; $r$ et $g$ sont les taux de recombinaison bande à bande et de dépiégeage; $N$ est la densité des pièges normalement vides ; $\left(N-n_{1}\right) S v$ est le taux de piégeage par les $\left(N-n_{1}\right)$ centres vides; $S$ est la section efficace de capture des électrons et $v$ leur vitesse; $l$ est le taux de création de paires électrons-trous :

$$
l=\beta K I_{0} \mathrm{e}^{-K x}
$$

$K$ est le coefficient d'absorption, $I_{0}$ le nombre de photons incidents par $\mathrm{cm}^{2}$ par $\mathrm{s}, x$ la distance à la surface de l'échantillon, $\beta$ le nombre de paires électrons-trous créées par un photon.

Aux temps très courts (partie $\mathrm{AB}$ des courants transitoires), l'équation (1) devient :

$$
\frac{\mathrm{d} n}{\mathrm{~d} t}=l-n(r+N S v)
$$

dont la solution est :

$$
\begin{aligned}
& n=\frac{l}{r+N S v}[1-\exp (-(r+N S v) t)] \\
& n=n_{0}\left(1-\mathrm{e}^{-t / \tau}\right)
\end{aligned}
$$

où le temps de vie des porteurs et leur densité à l'équilibre sont définis par :

$$
\tau=\frac{1}{r+N S v} \quad \text { et } \quad n_{0}=\frac{l}{r+N S v}=\tau l \text {. }
$$


Dans le cas où la pénétration des photons est très inférieure à l'épaisseur de l'échantillon, le courant total traversant l'échantillon est [11] :

$$
\Delta i=\beta \tau i_{0} e\left(\mu^{+}+\mu^{-}\right) E W\left(1-\mathrm{e}^{-t / \tau}\right)
$$

$E$ est le champ électrique appliqué; $W$ est la largeur de l'électrode. La durée de vie $\tau$ des porteurs ne peut être mesurée directement car elle est inférieure aux temps de réponse de l'appareillage; mais il est cependant possible de donner une valeur de $\mu \tau$ en supposant $: \mu=\mu^{+}=\mu^{-}$et $\beta=1$.

$$
(\Delta i)_{0}=2 \tau e \mu E I_{0} W
$$

soit :

$$
\mu \tau=\frac{(\Delta i)_{0}}{2 e E I_{0} W}
$$

Pour un échantillon n'ayant jamais été chauffé ni mis en présence d'oxygène, donc essentiellement de type cis, on trouve $\mu \tau=3 \times 10^{-11} \mathrm{~cm}^{2} / \mathrm{V}$. Cette valeur doit être considérée comme une valeur limite à cause de la surévaluation de $\beta(\beta=1)$. Elle peut varier légèrement d'un échantillon à l'autre. Elle est à comparer avec celle trouvée par Tani et al. [3] sur le trans-(CH) $)_{x}$ pur : $2 \times 10^{-10} \mathrm{~cm}^{2} / \mathrm{V}$. Mais ces auteurs ont calculé $\mu \tau$ à partir de mesures globales, ne permettant pas de distinguer les effets bande à bande des effets secondaires de piégeage. Rappelons enfin que dans le cas du silicium cristallin, $\mu \tau$ peut atteindre la valeur $\mu \tau=1 \mathrm{~cm}^{2} / \mathrm{V}$ [12] tandis que pour le Si amorphe, $\mu \tau=10^{-7} \mathrm{~cm}^{2} / \mathrm{V}$ [13].

Aux temps longs, $n$ a atteint sa valeur d'équilibre $n_{0}$ et l'équation (2) devient :

$$
\frac{\mathrm{d} n_{1}}{\mathrm{~d} t}=-\left(g+n_{0} S v\right) n_{1}+n_{0} N S v
$$

Son intégration donne :

$$
n_{1}=\frac{n_{0} N S v}{g+n_{0} S v}\left[1-\exp \left(-\left(g+n_{0} S v\right) t\right)\right]
$$

On pose

$$
g+n_{0} S v=1 / \tau_{\mathrm{p}}
$$

Si l'absorption de la lumière est importante, on peut remplacer la distribution $n_{0}(x)$ par une distribution homogène, égale à $0,37 \tau \beta K I_{0}$ sur une profondeur égale à $1 / K$. Si $g \ll n_{0} S v$, alors l'augmentation de courant due au piégeage est :

$$
\begin{aligned}
& \Delta i=V \int_{0}^{1 / K} N\left(1-\mathrm{e}^{-t / \tau_{\mathrm{p}}}\right) \frac{W}{L} e \mu^{+} \mathrm{d} x \\
& \Delta i=\frac{V}{L} \frac{W}{K} N e \mu^{+}\left(1-\mathrm{e}^{-t / \tau_{\mathfrak{p}}}\right) .
\end{aligned}
$$

Ceci correspond à la partie BC des courants transitoires. Ce photocourant doit être de type exponentiel (du moins tant que l'on considère un seul type de pièges ou des pièges caractérisés par des taux de piégeage très distincts) de constante de temps $\tau_{\mathbf{p}}$.

Il se sature à une valeur $(\Delta i)_{\mathrm{s}}=\frac{W}{K} \frac{n_{0} N S v}{g+n_{0} S v} e \mu^{+} E$ pratiquement indépendante du flux 
lumineux et approximativement égale à $N e \mu^{+} E \frac{W}{K}$. Cette saturation est atteinte plus ou moins rapidement suivant la valeur de ce même flux lumineux, car $\tau_{\mathrm{p}}$ dépend de $n_{0}$.

Pour certains échantillons, ou quand on utilise des filtres lumineux à très large bande, cette augmentation est parfois cachée par les réponses de l'appareillage et est confondue avec l'accroissement rapide AB. Il faut alors diminuer le flux lumineux pour détecter l'effet de piégeage. En introduisant la valeur $n_{0}$ dans (3) on a :

$$
1 / \tau_{\mathbf{p}}=g+0,37 \tau \beta K S v I_{0} .
$$

La courbe représentant $1 / \tau_{\mathrm{p}}$ en fonction de $I_{0}$ est une droite de pente $0,37 \beta \tau S v K$ dont l'ordonnée à l'origine est $g$ (Fig. 2). D'autre part :

$$
\frac{(\Delta i)_{s}}{\tau_{\mathrm{p}}}=0,37 \times \frac{V}{L} W N e \mu \tau S v I_{0} .
$$

La pente à l'origine du photocourant dans sa partie $\mathrm{BC}$ varie linéairement ávec le flux lumineux (Fig. 3).

On peut calculer une valeur du taux de dépiégeage $g$ voisine de $5 \times 10^{2} \mathrm{~s}^{-1}\left(\tau_{\mathrm{p}}=1 / g=1 \mathrm{~s}\right.$ dans le cas du Si type p) [14].

Lorsque l'échantillon est remis dans l'obscurité, ces porteurs dépiégés ont beaucoup plus de chance de se recombiner que de tomber à nouveau dans un piège.

Dans ces conditions, l'équation (2) se réduit à :

$$
\frac{\mathrm{d} n_{1}}{\mathrm{~d} t}=-g n_{1} \quad \text { d'où } n_{1}=N_{1} \exp \left(-\frac{t}{\tau_{\mathrm{p}}}\right)
$$

où $N_{1}$ représente le nombre de pièges remplis à l'instant de la remise de l'échantillon dans le noir.

Les courbes obtenues sur différents échantillons ne présentent pas cette forme simple. Cependant, l'équation (4) est assez bien justifiée aux temps courts et effectivement les courbes des photo-

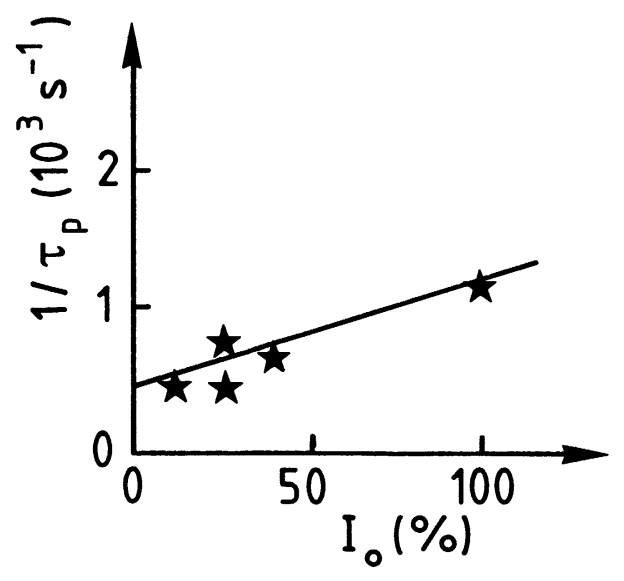

Fig. 2. - Variation de l'inverse de la constante de dépiégeage en fonction du flux lumineux incident exprimé en pourcents de la valeur maximum (irradiation à $\lambda=3500 \AA$ ).

[Variation of the inverse of the detrapping constant as a function of the incident illumination intensity expressed in percent of the maximum value (at $\lambda=3500 \AA)$.] 


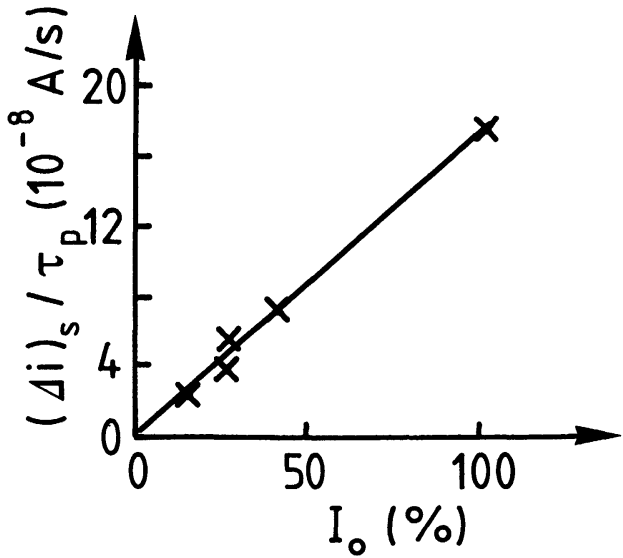

Fig. 3. - Variation de la pente à l'origine du photocourant en fonction du flux lumineux incident exprimé en pourcents de la valeur maximum (irradiation à $\lambda=3500 \AA$ ).

[Variation of the initial slope of the photocurrent as a function of the incident illumination intensity expressed in percent of the maximum value (at $\lambda=3500 \AA)$.]

courants aux temps courts sont de type exponentiel avec une constante de temps voisine de $5 \mathrm{~ms}$ (donc $g=1 / \tau_{\mathrm{p}}=2 \times 10^{2} \mathrm{~s}^{-1}$ ) ce qui confirme la valeur trouvée à partir des photocourants.

Il serait possible d'envisager une autre interprétation des résultats expérimentaux : la partie BC représentant un dépiégeage de porteurs majoritaires préalablement piégés. Cette hypothèse n'a pas été retenue notamment parce que l'excitation $(\lambda<3500 \AA)$ correspond à un mécanisme de création de porteurs bande à bande.

Quand le taux d'isomérisation cis-trans augmente, les deux composantes des photocourants sont de plus en plus difficiles à séparer, et de ce fait une interprétation simple devient plus délicate. D'autre part, une lumière de longueur d'onde plus grande (6 $250 \AA-2 \mu)$ devient active.

Le produit $\mu \tau$ est plus grand pour le trans- $(\mathrm{CH})_{x}$. Mais ceci peut être dû soit à une augmentation de $\tau$, soit à une augmentation forte de $\mu$ et une faible diminution de $\tau$. Dans le premier cas, l'hypothèse émise par Etemad et al. [2] selon laquelle la photoconduction passe par la création de solitons et d'antisolitons serait vérifiée. Mais ceci va à l'encontre des récents résultats obtenus sur le trans $-(\mathrm{CH})_{x}$ où l'étude du temps de vol des porteurs de charge $\left(<10^{-9} \mathrm{~s}\right)$ met en doute la relaxation des électrons et trous en solitons et antisolitons mobiles [15]. Cependant ces résultats concernent le polyacétylène entièrement isomérisé pour lequel la concentration de pièges est très importante.

Si $\mu \tau$ est de l'ordre de $10^{-10} \mathrm{~s}$ et si on adopte pour la valeur déterminée par Yacoby, Roth et al. [15] soit $\tau=10^{-12} \mathrm{~s}$, alors on trouve des mobilités de l'ordre de $10^{2} \mathrm{~cm}^{2} / \mathrm{V}$.s, ce qui est plausible pour une mobilité le long d'une chaîne.

En conclusion, nous avons montré que le mécanisme de transport des charges photocréées dans le polyacétylène est gouverné par le taux de recombinaison des porteurs et par leur piégeage. Ces deux processus sont faciles à séparer dans le cas du polyacétylène essentiellement cis. Des valeurs du produit $\mu \tau$ et du taux de dépiégeage ont été calculées.

Le comportement du trans- $(\mathrm{CH})_{x}$ est plus complexe. Des études mettant en ouvre des techniques de mesures plus rapides permettront de préciser les mécanismes de conduction dans des échantillons d'isomérisation cis-trans croissante. 


\section{Bibliographie}

[1] Lauchlan, L., Etemad, S., Chung, T. C., Heeger, A. J., McDiarmid, A. G., Phys. Rev. B 24 (1981) 3701.

[2] Etemad, S., Mitani, T., Ozaki, M., Chung, T. C., Hegger, A. J., McDiarmid, A. G., Solid State Commun. 40 (1981) 75.

[3] Tani, T., Grant, P. M., Gill, W. D., Street, G. B., Clarke, T. C., Solid State Commun. 33 (1980) 499.

[4] Guillaud, G., Maitrot, M., Davenas, J., Xu, X. L., Mathis, C., François, B., Andre, J. J., J. Physique Colloq. 44 (1983) C3-101.

[5] Sethna, J. P., Kivelson, S., Phys. Rev. B 26 (1982) 3513.

[6] Su, W. P., Schrieffer, J. R., Proc. Nat. Acad. Sci. 77 (1980) 5626.

[7] Etemad, S., Ozaki, M., Heeger, A. J., McDiarmid, A. G., Chem. Scr. 17 (1981) 159.

[8] Ito, T., Shirakawa, H., Ikeda, S., J. Polym. Sci. Polym. Chem. Ed. 13 (1975) 1943.

[9] Davenas, J., Xu, X. L., Maitrot, M., Gamoudi, M., Guillaud, G., Andre, J. J., François. B., Mathis, C., J. Physique Colloq. 44 (1983) C3-183.

[10] Haynes, J. R., Hornbeck, J. A., Phys. Rev. 100 (1955) 606.

[11] Ryvkin, S. R., Photoelectric effects in semiconductors (Consultant Bureau, N.Y.) 1964, p. 18.

[12] Petitclerc, A., Electronique Physique des semiconducteurs (Ed. du Tambourinaire, Paris) 1962.

[13] Oкамото, H., Kida, H., Nonomma, S., Fukumoto, K., Hamakawa, Y., J. Appl. Phys. 54 (1983) 3236.

[14] Hornbeck, J. A., Haynes, J. R., Phys. Rev. 97 (1955) 311.

[15] Yacoby, Y., Roth, S., Menke, K., Keilmann, F., Kuhl, J., Solid State Commun. 47 (1983) 869. 